\title{
La Enseñanza de la Tecnología Electrónica Incorporando las Hojas de Datos de los Fabricantes
}

\author{
A. Menéndez, C. León y A. López \\ Universidad de Sevilla, E.U.P., Departamento de Tecnología Electrónica, C/Virgen de África 7, \\ 41011 Sevilla-España
}

\begin{abstract}
Resumen
Se propone una metodología de enseñanza para cursos de tecnología electrónica. El método incluye un adecuado balance entre los conceptos básicos necesarios y la información entregada en hojas de datos y catálogos de los fabricantes. Usando este nuevo enfoque, se analiza la enseñanza de conceptos y aplicaciones de diodos semiconductores de rectificación. La metodología ha mostrado ser efectiva y debería ser incorporada en textos de tecnología electrónica.
\end{abstract}

Palabras clave: tecnología electrónica, componentes electrónicos, enseñanza-aprendizaje, componentes semiconductores

\section{Electronic Technology Teaching Using Manufacturer's Datasheets}

\begin{abstract}
A teaching methodology for electronic technology courses is proposed. The method includes an appropriate balance between the necessary basic concepts and the information provided in the manufactures datasheets and catalogs. Using this new approach, the teaching of concepts and applications of rectification superconductor diodes is analyzed. The methodology has shown to be effective and should be included in electronic technology books.
\end{abstract}

Keywords: electronic technology, electronic devices, teaching-learning process, semiconductor devices 


\section{INTRODUCCION}

Los componentes en general, y muy específicamente los dispositivos de estado sólido, han alcanzado un elevado nivel de perfeccionamiento e importancia económica. Este nivel desarrollo alcanzado por la industria fabricante de componentes electrónicos hace que el comportamiento de dichos componentes, caracterizados por las correspondientes Hojas de Datos del fabricante, resulte cada vez más distante de lo que podría ser un comportamiento tipo, deducido directamente de una comprensión del funcionamiento básico del dispositivo.

Desde el punto de vista del "utilizador" de componentes (como podría ser el caso de un diseñador de circuitos), frente a la penosa alternativa de una amplia comprensión del funcionamiento interno de toda una serie de dispositivos a partir de la comprensión de la correspondiente Hoja de Datos, en base a un conocimiento elemental del funcionamiento del componente.

En consecuencia, en el presente artículo se plantea el interés de orientar la enseñanza de la Tecnología Electrónica hacia una serie de conocimientos que permitan establecer el puente entre la comprensión de unos fundamentos básicos y la interpretación de Hojas de Datos de los correspondientes componentes.

El presente artículo expone, a título de ejemplo, un ejercicio básico de rectificación con diodos semiconductores, orientado con este enfoque de la enseñanza de la Tecnología Electrónica conocimientos básicos, características prácticas y resolución de ejercicios con componentes reales.

\section{APLICACIÓN A LA RECTIFICACIÓN DE MEDIA ONDA}

En la aplicación de rectificación de media onda que aquí se desarrolla, se pretende en primer lugar determinar las condiciones operativas a partir del conocimiento de las características del diodo. En segundo lugar se plantea la estimación de la temperatura del diodo, con la cual se podrán modificar eventualmente las condiciones operativas anteriores.

Estimadas dichas condiciones operativas, se verificará que estas son compatibles con los valores limites de funcionamiento del diodo. En caso positivo, se procederá a seleccionar el modelo de diodo adecuado según las tensiones inversas de trabajo utilizadas.

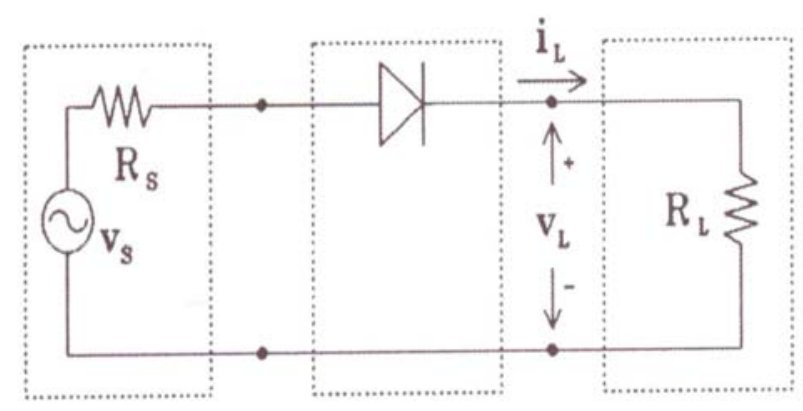

Fig. 1: Circuito rectificador de media onda

\section{Estudio Teórico}

Para rectificador de media onda (Figura 1) considerado (Gualda et al., 1992 y Malvino, 1994), se evaluaron los siguientes aspectos:

1.- Comportamiento en directa o en inversa: Cuando la tensión que suministra la fuente es positiva y superior a la tensión umbral del diodo, este conduce, siendo la tensión en el diodo ( $V_{D}>$ $\vee \gamma$ ) y en la carga proporcional a la de la fuente: 


$$
\begin{aligned}
& v_{D}=v_{S} \frac{R_{O N}}{R_{S}+R_{O N}+R_{L}}+V_{\gamma} \\
& v_{L}=\left(v_{S}-V_{\gamma}\right) \frac{R_{L}}{R_{S}+R_{O N}+R_{L}}
\end{aligned}
$$

donde Ron es la resistencia del diodo en conducción

Por otro lado, la corriente será:

$$
i_{D}=i_{L}=\frac{v_{S}-V_{\gamma}}{R_{S}+R_{O N}+R_{L}}
$$

Si $v_{S}$ es negativa, el diodo estará en corte y la única corriente que circulará por el será la de saturación inversa:

$$
\begin{aligned}
& i_{D}=i_{L}=-I_{0} \\
& v_{L}=-I_{0} \times R_{L}
\end{aligned}
$$

2.- Limitación por tensión máxima: La tensión máxima en la carga, $V_{L}$, se obtiene cuando el diodo está en conducción y depende de la amplitud de la tensión de la fuente, $V_{S}$, y de $V_{\gamma}$. Cuando $V_{s}>V_{\gamma}$, se puede despreciar el efecto de $V_{\gamma}$ y el diodo no ejerce ninguna limitación.

$v_{D}=-v_{S}+I_{0}\left(R_{S}+R_{L}\right)$

Por otro lado, cuando el diodo no conduce, toda la tensión de la fuente cae en el diodo, limitando la tensión inversa de este el valor máximo de $V_{S}$. Como este proceso se repite cada ciclo de la señal de entrada, la magnitud que hay que considerar es la tensión inversa de pico repetitiva, $V_{R M M}$.

3.- Limitación por corriente máxima: La máxima circulación de corriente en la carga se produce cuando el diodo está en conducción. Como esta intensidad es la misma que circula por el diodo, la limitación vendrá impuesta por la máxima corriente directa que es capaz de soportar el diodo:

$$
I_{L \max }=I_{F \max }=\frac{V_{S} \times V_{\gamma}}{R_{S}+R_{O N}+R_{L}}
$$

Por idéntico razonamiento que en el apartado anterior, la magnitud concreta a tener en cuenta es la corriente directa de pico repetitivo, $I_{F R M}$.

4.- Potencia disipada en el diodo: La potencia disipada en el diodo durante su conducción es aproximadamente, la indicada por la siguiente fórmula.

$$
P_{\text {DIR media }} \approx \frac{V_{S} \times V_{\gamma}}{\Pi \times\left(R_{S}+R_{O N}+R_{L}\right)}
$$


Para llegar a este resultado se ha tenido en cuenta que $V_{S} \gg V_{\gamma}$ y se ha despreciado el efecto de $R_{O N}$ frente al valor de la carga.

En el caso de polarización inversa del diodo, el resultado es:

$P_{I N V \text { media }} \approx \frac{V_{S} \times I_{0}}{\Pi}$

5.- Temperatura del diodo: La temperatura de funcionamiento del diodo se calcula:

$T=T_{A}+R_{t h} \times P$

Suponiendo la alimentación a $50 \mathrm{~Hz}$, el período es suficientemente pequeño para que se pueda calcular la temperatura media del diodo utilizando la potencia media según la expresión:

$P=\frac{1}{2}\left(P_{\text {DIR media }}+P_{\text {INV media }}\right)$

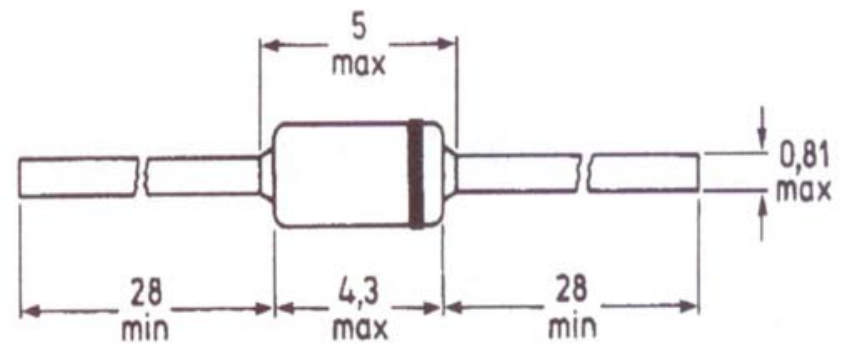

Fig. 2: Diodo rectificador de silicio IN4001

Hoja de Datos

En esta aplicación se plantea la utilización de u diodo de la serie IN4000 (Phillips, 1992) cuyas características aparecen resumidas en las Figuras 2, 3 y 4 en la Tabla 1.

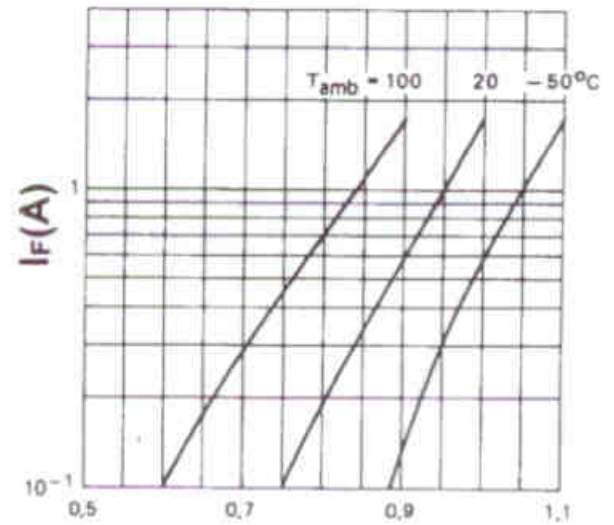

Fig. 3: Característica V-I típica del diodo IN4001

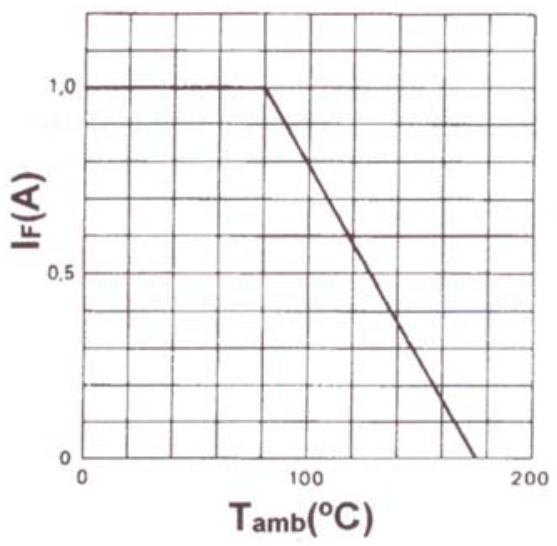

Fig. 4: Corriente máxima en el diodo en función de la temperatura ambiente 


\section{Obtención de resultados}

Se considera que la fuente suministra $36 \mathrm{Vef}_{\text {ef }} 50 \mathrm{~Hz}$, y que su resistencia interna es de $1.5 \Omega$. Suponemos además que la carga vale $15 \Omega$ y la temperatura ambiente $25^{\circ} \mathrm{C}$. Se pretende determinar el diodo más adecuado para el diseño del rectificador dentro de la serie IN4000.

Tabla 1: Características del yodo IN4001 (tomado de Phillips, 1992)

\begin{tabular}{|c|c|c|c|c|}
\hline \multicolumn{5}{|c|}{ RATINGS: Limiting values in accordance with the Absolute Maximum Systems (IEC 134) } \\
\hline & $1 \mathrm{~N} 4001 \mathrm{ID}$ & 4002ID & 4003ID & $4004 \mathrm{ID}$ \\
\hline Repetitive peak reverse voltage $V_{R B M} \max$. & 50 & 100 & 200 & 400 \\
\hline $\begin{array}{c}\text { Continous reverse voltage } \\
V_{R} \max .\end{array}$ & 50 & 100 & 200 & 400 \\
\hline $\begin{array}{l}\text { Average forward current (average over any } \\
20 \mathrm{~ms} \text { period) } \\
\text { up to } \mathrm{T}_{\text {amb }}=75^{\circ} \mathrm{C} \\
\text { at } \mathrm{T}_{\text {amb }}=100^{\circ} \mathrm{C}\end{array}$ & \multicolumn{4}{|c|}{$\begin{array}{cc}\mathrm{I}_{\mathrm{FAV}} \max . & 1.0 \mathrm{~A} \\
\mathrm{I}_{\mathrm{F}(\mathrm{A})} \max . & 0.75 \mathrm{~A}\end{array}$} \\
\hline Repetitive peak forward current & \multicolumn{4}{|c|}{$I_{\mathrm{FRM}} \max .10 \mathrm{~A}$} \\
\hline $\begin{array}{l}\text { Non-repetitive peak forward current } \\
\text { (half-cycle sinewave, } 60 \mathrm{~Hz} \text { ) }\end{array}$ & \multicolumn{4}{|c|}{$\mathrm{I}_{\mathrm{FSM}} \max .20 \mathrm{~A}$} \\
\hline Storage temperature & \multicolumn{4}{|c|}{$\mathrm{T}_{\text {ute }}-65$ to $+175^{\circ} \mathrm{C}$} \\
\hline Junction temperature & \multicolumn{4}{|c|}{$\mathrm{T}_{i} \max .175^{\circ} \mathrm{C}$} \\
\hline \multicolumn{5}{|c|}{ THERMAL RESISTANCE } \\
\hline $\begin{array}{l}\text { Thermal resistance } \\
\text { from junction to ambient; device mounted on } \\
\text { a 1,5 mm thick epoxy-glass printed circuit } \\
\text { board; Cu thickness } \geq 40 \mu \mathrm{m}\end{array}$ & \multicolumn{4}{|c|}{$R_{\text {th } j-\mathrm{a}}=120 \mathrm{~K} / \mathrm{W}$} \\
\hline \multicolumn{5}{|c|}{ CHARACTERISTICS: $\mathrm{T}_{\mathrm{amb}}=25^{\circ} \mathrm{C}$ unless otherwise stated } \\
\hline $\begin{array}{c}\text { Forward voltage } \\
\mathrm{I}_{\mathrm{F}}=1 \mathrm{~A}\end{array}$ & \multicolumn{4}{|c|}{$\mathrm{V}_{\mathrm{F}}<1,1 \mathrm{~V}$} \\
\hline $\begin{array}{l}\text { Full-cycle average forward voltage } \\
\qquad \mathrm{I}_{\mathrm{FAa}}=1 \mathrm{~A}\end{array}$ & \multicolumn{4}{|c|}{$\mathrm{V}_{\mathrm{FAN}}<0,8 \mathrm{~V}$} \\
\hline $\begin{array}{c}\text { Reverse current } \\
V_{R}=V_{R \max } \\
V_{R}=V_{R \max } ; T_{\text {amb }}=100^{\circ} \mathrm{C}\end{array}$ & \multicolumn{4}{|c|}{$\begin{array}{l}I_{R}<10 \mu \mathrm{A} \\
I_{R}<50 \mu \mathrm{A}\end{array}$} \\
\hline
\end{tabular}

Teniendo en cuenta que el valor máximo de tensión a la entrada del rectificador es $50.9 \mathrm{~V}$., se deduce que sería suficiente con seleccionar el dispositivo IN4002, con objeto de que no se supere la tensión inversa de pico repetitivo $V_{R R M}$.

La corriente directa media que soporta el diodo es el parámetro $I_{F(A V)}$ y vale $1.0 \mathrm{~A}$. para toda la serie. La corriente máxima viene determinada por $I_{F R M}$, valiendo $10 \mathrm{~A}$. Para las características indicadas del circuito, la máxima intensidad que circula es aproximadamente $3.08 \mathrm{~A}$. según (7), calculado por exceso al haber despreciado $V_{\gamma}$ y $R_{O N}$. Este valor está por debajo de las limitaciones del diodo seleccionado.

Para el cálculo de la potencia directa disipada se necesita conocer la resistencia en conducción $R_{O N}$ y la tensión umbral $V_{\gamma}$ del diodo. Se calcula $R_{O N}$ como $\Delta V_{F} / \Delta I_{F}$, a partir de la Figura 3. Previamente, se necesita conocer el valor medio de la corriente en el diodo: 


$$
I_{F}(A v)=\frac{I_{F \max }}{\Pi}\langle 0.98 A
$$

Tomando los puntos comprendidos entre 1 y $0.6 \mathrm{~A}$ de la Figura 3 , se observa que $V_{F}$ oscila entre 0.95 y .09 , por tanto, $R_{O N} \approx 0.125 \Omega$

De la Figura 3 se obtiene un punto medio de funcionamiento del diodo, caracterizado por $I_{F}=$ 0.98 A., $V_{F}=0.95 \mathrm{~V}$. Extrapolando, según la pendiente dada por $R_{O N}$, se obtiene $V_{\gamma}$ aproximadamente igual a $0.8 \mathrm{~V}$. Con ello se deduce $P_{\text {DIR media }}=0.75 \mathrm{~W}$, a partir de (8).

Para calcular la potencia en inversa hace falta conocer la corriente inversa $I_{0}$, que según la Tabla 1 puede considerarse como $10 \mu \mathrm{A}$. Esto da una potencia disipada en inversa (9) entorno a 162 $\mu \mathrm{W}$, lo cual resulta claramente muy inferior a la disipada en directa y de efecto despreciable.

Para evaluar la temperatura en el diodo se utiliza la resistencia térmica, $R_{t h}$, dada en la Tabla $1 \mathrm{y}$ de valor $120 \mathrm{~K} / \mathrm{W}$. Con ello se obtiene $73^{\circ} \mathrm{C}$, empleando la ecuación (10). Con este incremento de temperatura se observa en la Figura 3, que las condiciones operativas se ven afectadas en la tensión de codo, que diminuiría en $0.1 \mathrm{~V}$ aproximadamente. Sin embargo, la corriente máxima que puede soportar el diodo no cambia (Figura 4), con lo cual, se concluye que, en primera aproximación, este efecto puede despreciarse.

\section{CONCLUSIONES}

El ejemplo tratado expone claramente la contribución del manejo directo de una Hoja de Datos como fuente de información sobre componentes reales y como elemento didáctico en sus aspectos de: interpretación de características prácticas y selección de componentes.

El interés del manejo directo de Hojas de Datos puede reforzarse notablemente si, además, los mismos componentes se utilizan para desarrollar pequeños trabajos de simulación y/o prácticas de laboratorio, que permitan contrastar los resultados previamente obtenidos mediante ejercicios del tipo aquí tratado.

Finalmente, se desea también señalar que, según la experiencia de los autores (Menéndez, 1995), el proyecto docente en Tecnología Electrónica resulta todavía más reforzado si el estudiante participa en búsqueda y selección de Hojas de Datos que reflejan el estado del mercado de componentes electrónicos.

\section{REFERENCIAS}

Gualda, J.A., S. Martínez y J.A. Martínez; Electrónica Industrial: Técnicas de Potencia. Marcombo (1992).

Malvino, A.P.; Principios de Electrónica. McGraw Hill (1994).

Menéndez, A.; Actividades complementarias en la enseñanza de la Tecnología Electrónica. III Jornadas Universitarias sobre innovación educativa en las Enseñanzas Técnicas, Ferrol, (1995).

Phillips Semiconductors. Discrete Semiconductors. Diodes. Data Hanbook SC01. (1992). 